\title{
VisDa: Creación de un plugin para WordPress
}

Ignasi Alcalde Tamer Al-kassimi Josep Gassó Julià Minguillón Enric Rodríguez 


\section{VisDa: creación de un plugin para WordPress}

Grupo de trabajo formado por: Ignasi Alcalde, Tamer Al-kassimi, Josep Gassó, Julià Minguillón, Enric Rodríguez

\section{Introducción}

Este documento es el resultado del taller organizado por Mosaic para la creación de un plugin para WordPress (WP), con el doble objetivo de aprender a crear este tipo de herramientas y el de mejorar las funcionalidades de la propia revista Mosaic.

Como resultado del taller VisDa: creación de un plugin para WordPress se formaron dos grupos de trabajo. El resultado del primer grupo de trabajo ya se publicó en enero de 2012: http://mosaic.uoc.edu/2012/01/30/visda-creacion-de-un-plugin-para-wordpress/.

El segundo grupo de trabajo desarrolló otro plugin de visualización de datos, el resultado del cual se presentará en estas páginas.

La formación de un grupo heterogéneo de profesionales y estudiantes se tradujo en una experiencia enriquecedora. Fue precisamente la multidisciplinaridad de los componentes del grupo lo que convirtió al taller en algo tan positivo e interesante, lo cual permitió combinar diferentes disciplinas y competencias que van desde la programación a la analítica y arquitectura de información.

\section{Estructura y objetivos del Workshop}

El taller se desarrolló presencialmente en dos sábados. En la primera sesión se formaron dos grupos de trabajo, se estableció la cronología de desarrollo del plugin y se crearon los accesos a sendos espacios de trabajo compartido mediante BSCW.

El objetivo final del workshop era crear un plugin mediante el cual representar visualmente datos obtenidos de la base de datos de WordPress y hacerlos inteligibles para el usuario.

La primera sesión comenzó con la entrega y presentación de diferentes documentos que servirían como base para la creación del plugin.

a. Visualización de la información, por Laia Blasco.

b. Codificar en y para WordPress (PHP), por César Córcoles.

c. La base de datos de WordPress (MySQL), por Carlos Casado.

d. Processing y processing.js, por Quelic Berga. 


\section{Preparación de los datos: qué presentar visualmente}

Una vez realizadas las introducciones y presentaciones pertinentes, quedaba por decidir qué información presentar visualmente. Se pretendía crear un plugin que representara información útil. Esta mención puede parecer banal pero no lo es, puesto que también se podría presentar información simplemente con fines visuales y artísticos.

En una hoja se plasmaron todos los tipos de datos con los que se podía trabajar (información obtenida en la explicación de "la base de datos de WordPress (MySQL)" de Carlos Casado). EI azar y la mano inocente de Quelic Berga quisieron que en el plugin se cruzaran los siguientes tipos de datos: número de visitas, tags, categorías y fechas de publicación.

Si bien el uso de estos datos no era cerrado ni obligatorio, sirvió de buen punto de partida para realizar los planteamientos gráficos y proceder al posterior desarrollo.

\section{A) Primera propuesta}

La primera propuesta presentaba una estructura visual horizontal y una navegación lineal en dos pantallas:

- La primera pantalla mostraba una red de tags enlazados entre ellos. Dos tags están relacionados si son usados a la vez en un mismo post al menos un cierto número de veces. El tamaño de las esferas definía la cantidad de visitas. Éstas eran clicables, de manera que se accedía a la segunda pantalla.

- La segunda pantalla mostraba el tag seleccionado y una relación de las entradas de la revista ordenadas cronológicamente (de más a menos recientes) y separadas por categorías.

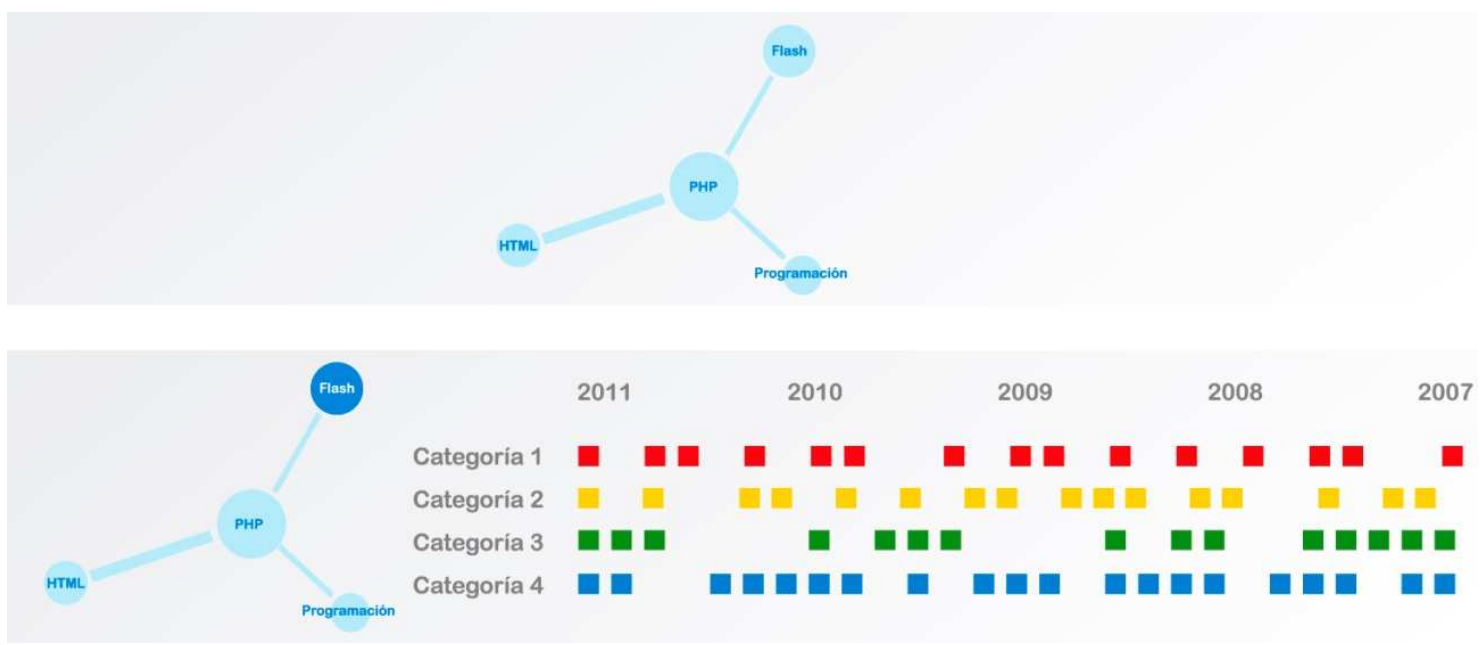


Esta propuesta se desestimó por parecerse en exceso a una de las muestras que se habían presentado durante la introducción del workshop.

\section{B) Segunda propuesta}

En la segunda propuesta se pasó de una disposición de la información horizontal a una disposición circular. Con respecto a la primera propuesta se mantenía la navegación en dos pantallas y la presentación de la red de tags en la primera.

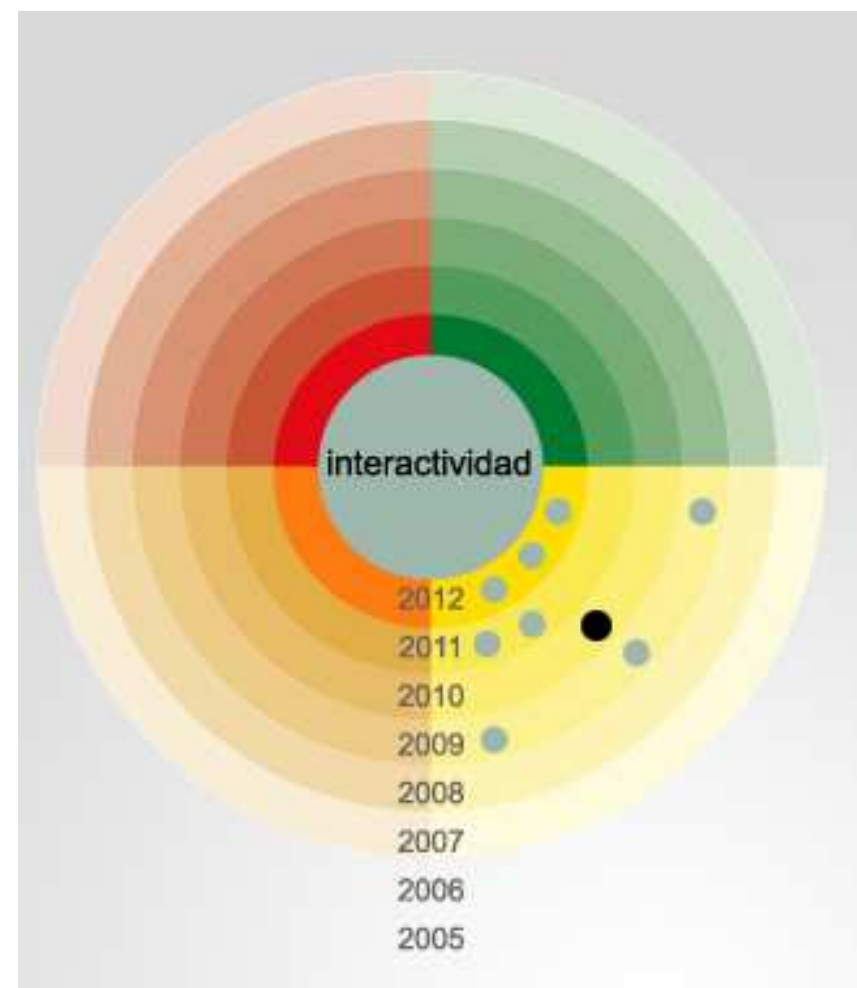

VisDa: creación de un plugin para WordPress 26/07/2011 Joan Ferrater i Olaya

En esta propuesta se redistribuyó la información de la segunda pantalla. Como se aprecia en la imagen, se propuso una disposición similar a la de una diana, en la que el radio representaba la cronología y los arcos definían las categorías, que en el caso que nos ocupaba eran las cuatro principales de la revista Mosaic (recursos -rojo-, entrevistas -verde-, artículos -amarillo-, experiencias -naranja-). En el centro se presentaba la etiqueta que el usuario había seleccionado. 
Esta propuesta se desestimó por varios motivos:

- La disposición de los puntos en los arcos hacía que, al alejarse en el tiempo, la cantidad de entradas pareciera inferior en comparación con el mismo número de entradas en un año más reciente.

- Con vistas a posteriores evoluciones, permitir al administrador de WordPress seleccionar la cantidad de categorías podría dificultar extremadamente la programación con processing.

- La percerpción circular focaliza la mayor atención en el centro, dando margen de error y mas peso visual a las entradas mas recientes.

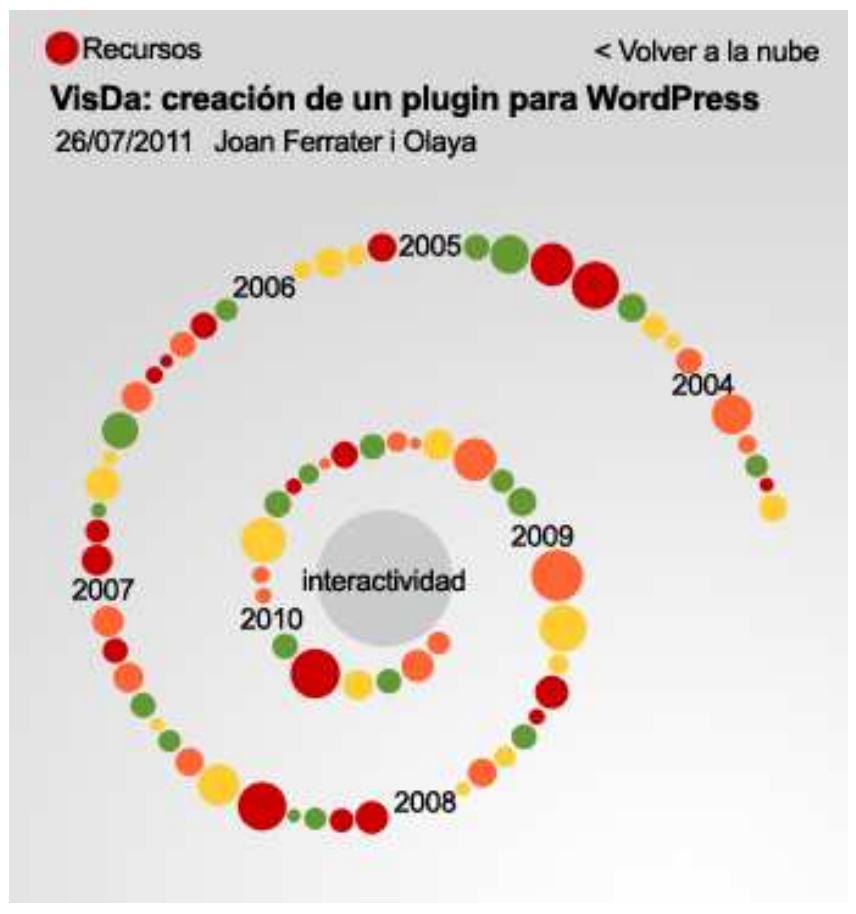

\section{C) Tercera propuesta}

Finalmente se creó una tercera propuesta que sería la que finalmente se desarrollaría.

En ésta se creó una línea de tiempo que simulaba la cronología y se le dio forma de espiral de manera que se mantenía la continuidad en el tiempo y el espacio ocupado era moderado. Al igual que en la propuesta anterior, el tag seleccionado por el usuario quedaba centrado en el marco visual. 


\section{Desarrollo del plugin}

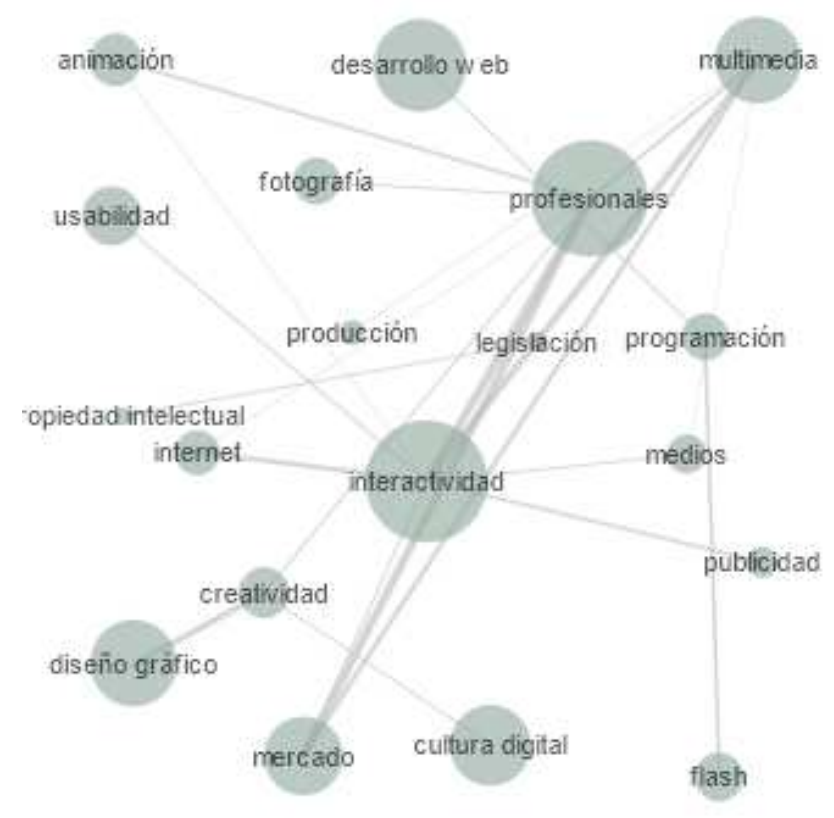

Con la imagen gráfica consensuada y aprobada por el grupo se procedió a la programación del plugin. Contar en el equipo con un programador experimentado facilitó con creces el desarrollo. Processing no resultó ser un lenguaje complejo, pero no está de más conocer algunas de las vicisitudes que entraña programar con éste.

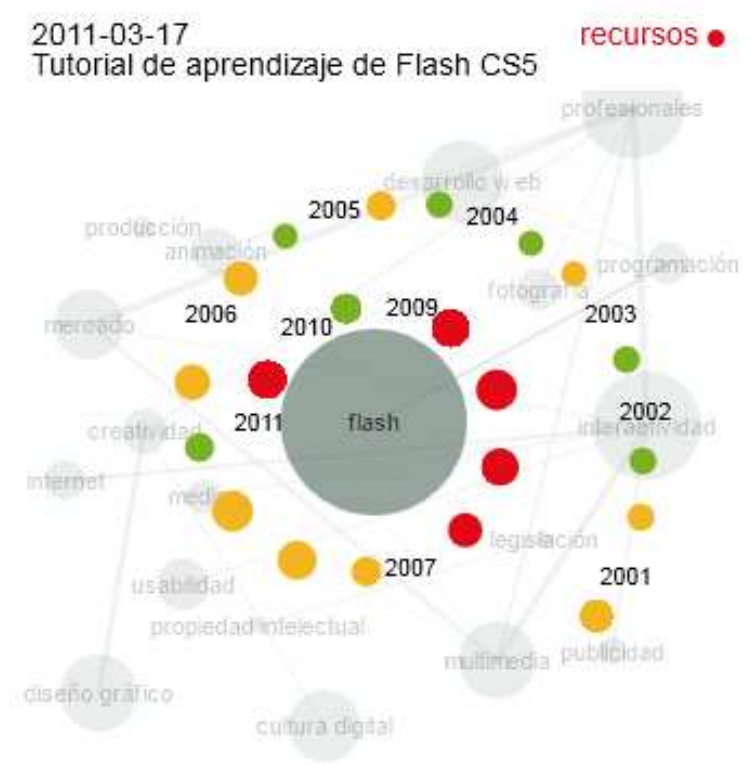




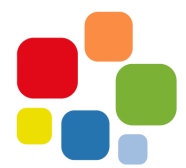

Por otra parte, processing.js también introdujo ciertas complicaciones adicionales que fueron solucionadas estudiando el código fuente original del mismo para entender cómo funciona internamente y cómo se implementan ciertas funciones básicas para el éxito del plugin.

La fase de desarrollo que comenzó con processing y debía acabar con la integración con WP mediante PHP había costado más de lo que se presuponía, pero el resultado resultó satisfactorio y quedó abierto a margen de mejora.

\section{Instalación del plugin}

1. Descomprimir el archivo .zip del plugin en la carpeta wp-content/plugins.

2. Activarlo desde el administrador de WordPress.

3. Poner el shortcode correspondiente entre claudátors dentro del post dónde se quiera visualizar, [visda] en este caso.

\section{Propuestas de mejora}

Aunque el funcionamiento del plugin es bastante consistente, hay aspectos que podrían mejorarse en futuras versiones:

- Las opciones de configuración en el panel de control de WP son nulas. Podría añadirse un selector de categorías para que el administrador eligiera cuáles son susceptibles de ser gestionadas por el plugin. También se podría decidir la densidad de la red de tags, por ejemplo.

- Se han detectado algunas inconsistencias en diferentes navegadores y sistemas operativos. Sería conveniente analizarlas y detectar el origen de estos problemas para posteriormente aplicar las correcciones pertinentes.

\section{Conclusión}

Formar parte de un equipo con especialidades tan diversas es más que una experiencia. Al principio cuesta encontrar el sitio, pero finalmente se llega a la sensación de ser la pieza de un puzle. Es inevitable que algunas piezas sean más grandes que otras, pero todas ellas realizan aportaciones importantes para la consecución del proyecto.

La gestión del tiempo es quizás uno de los factores determinantes a la hora de realizar actividades de esta índole. Aunque el taller se enmarcaba en dos sesiones presenciales que apenas sumaban doce horas en total, la dedicación (tanto con reuniones presenciales como 
a distancia) fue mucho mayor. Quizás este fue uno de los pecados que algunos cometimos al inscribirnos al taller y que finalmente gestionamos como buenamente pudimos.

Sea como sea, la experiencia fue realmente enriquecedora y altamente recomendable. Si eres alumno y tienes la opción de participar en un workshop de esta clase, no lo dudes ni un segundo: todo el mundo puede aportar su granito de arena, se aprende mucho y, lo más importante, se conocen profesionales de diferentes ámbitos con visiones sobre un mismo tema muy complementarias. 